\title{
Dürfen, können, sollen Psychologische Psychotherapeuten Tätigkeiten übernehmen, die heute noch Ärzten vorbehalten sind?
}

Seit geraumer Zeit wird, angestoßen durch das Forschungsgutachten zum Psychotherapeutengesetz, in der Fachöffentlichkeit vermehrt die Frage diskutiert, ob auch Psychologische Psychotherapeuten (PP) und Kinder- und Jugendlichenpsychotherapeuten (KJP) Tätigkeiten übernehmen können, die bislang nur Ärzten vorbehalten sind. Dabei geht es im Wesentlichen um die Verordnung von Heil- und Arzneimitteln und um die Befugnis zur Ausstellung von Arbeitsunfähigkeitsbescheinigungen, zur Krankenhauseinweisung bzw. zur Überweisung an Fachärzte. In den beiden Beiträgen in diesem Pro und Contra werden die jeweiligen Positionen der betroffenen Berufsgruppen (Ärzte und PP/KJP) hierzu zusammenfassend gegenübergestellt. Die ärztliche Seite wird vertreten durch Frau Prof. Sabine Herpertz (Heidelberg), die der psychologischen Kolleginnen und Kollegen von Dipl.-Psych. Timo Harfst und Dr. phil. Johannes Klein-Heßling (Bundespsychotherapeutenkammer Berlin).

Da diese Diskussion von großer gesundheitspolitischer Bedeutung ist, sollten sich die Mitglieder der betroffenen ärztlichen und psychologischen Berufsgruppen hierzu eine Meinung bilden. Unsere Pro-Contra-Debatte soll einen Beitrag zu diesem Meinungsbildungsprozess leisten.

Fritz Hohagen, Lübeck

\section{+ Pro}

Ein Hausarzt ist zur Beurteilung der Arbeitsfähigkeit aufgrund einer psychischen Störung nicht besser qualifiziert als der behandelnde Psychotherapeut

Psychologische Psychotherapeuten (PP) und Kinder- und Jugendlichenpsychotherapeuten (KJP) sind durch ihre Approbation Fachärzten in der vertragsärztlichen Versorgung grundsätzlich gleichgestellt. Ausnahmen sind bisher die Befugnisse zur Verordnung von Heilmitteln (relevant sind hier Ergo-, Logo- und Soziotherapie) und Arzneimitteln, zur Ausstellung von Arbeitsunfähigkeitsbescheinigungen, zur Einweisung in die stationäre psychotherapeutische oder psychiatrische Behandlung und zur Überweisung an Fachärzte. Diese heilberuflichen Tätigkeiten dürfen PP und KJP bisher nicht übernehmen, eine Aufhebung dieser Befugniseinschränkungen von PP und KJP wäre aber sowohl möglich als auch nötig.
PP und KJP erwerben in ihrer staatlich geregelten Ausbildung (mit Ausnahme der Arzneimittelverordnungen) umfassende Kompetenzen für die Versorgung psychisch kranker Menschen. Ein Hochschulstudium und die anschließende Psychotherapeutenausbildung qualifizieren sie dazu, eigenverantwortlich Heilkunde auszuüben. Obligatorische praktische Erfahrungen in der stationären Versorgung psychisch kranker Menschen machen sie mit diesem Versorgungssetting und seinen Indikationen vertraut. In der praktischen Arbeit in multiprofessionellen Behandlungsteams erwerben sie durch die Erstellung von Behandlungsplänen, die auch andere Behandlungsmethoden als Psychotherapie umfassen, ausreichende Qualifikationen. Die in psychiatrischen und psychosomatischen Krankenhäusern und Rehabilitationskliniken beschäftigten PP und KJP werden mit dieser Aufgabe regelhaft beauftragt, ebenso wie mit der sozialmedizinischen Begutachtung von Patienten und der Planung und Koordination der weiteren ambulanten und gegebenenfalls auch stationären Behandlung nach Entlassung. Diese Versorgungsstrukturen

\section{KARGER}

Fax +497614520714

Information@Karger.de

www.karger.com (c) 2010 S. Karger GmbH, Freiburg 
und die anderen Behandlungsangebote einschließlich der Pharmakotherapie sind darüber hinaus Gegenstand der Staatsprüfung. PP und KJP sind damit fachlich ausreichend qualifiziert, eigenverantwortliche Entscheidungen zur Verordnung von Heilmitteln, zur Einweisung in ein Krankenhaus bzw. zur Überweisung an einen Facharzt zu treffen.

Als Experten für die Diagnostik und Behandlung psychischer Erkrankungen sind PP und KJP qualifiziert, die Schwere einer psychischen Erkrankung und das jeweilige Erkrankungsstadium zu bestimmen und eine fachlich fundierte Prognose des Krankheitsverlaufes abzugeben. Sie kennen die evidenzbasierten nichtpsychotherapeutischen Behandlungsmöglichkeiten einschließlich der medikamentösen Therapie und der Kombinationsbehandlung und kommen ihrer berufsrechtlichen Verpflichtung nach, den Patienten vor Beginn einer Behandlung auch über die evidenzbasierten Behandlungsalternativen zur Psychotherapie und ihre Erfolgsaussichten $\mathrm{zu}$ informieren. Sie erfüllen damit alle Anforderungen, um eine vorliegende Arbeitsunfähigkeit feststellen und attestieren zu können. Ferner sind sie in der Lage zu entscheiden, ob eine psychische Erkrankung so schwer ausgeprägt ist, dass akute Selbst- oder Fremdgefährdung vorliegt, ob für die Fortsetzung der ambulanten Behandlung eine ausreichende Absprachefähigkeit besteht oder ob eine stationäre Behandlung unmittelbar erforderlich ist.

Das reale Tätigkeitsspektrum von PP und KJP in den psychiatrischen und psychosomatischen Einrichtungen belegt, dass ihnen die für die Krankenhaus- bzw. Abteilungsleitung Verantwortlichen, abseits der berufspolitischen Diskussionen, diese Kompetenzen schon lange zuschreiben. Daher ist es fachlich nicht nachvollziehbar, warum im ambulanten Bereich formal stets ein Arzt konsultiert werden muss, der dazu mit dem Patienten möglicherweise zum ersten Mal in Kontakt tritt. Ein Hausarzt ist zur Beurteilung der Arbeitsfähigkeit aufgrund einer psychischen Störung nicht besser qualifiziert als der behandelnde Psychotherapeut.

Zur Verordnung von Psychopharmaka vermitteln das Hochschulstudium sowie die theoretische und praktische Ausbildung Grundkenntnisse, die allerdings für eine generelle Befugnis zur Verordnung von Arzneimitteln durch PP oder KJP noch nicht ausreichen. Im Rahmen einer angemessenen psychopharmakologischen Zusatzweiterbildung im Bereich der Psychopharmakologie einschließlich der relevanten Weiterbildungsinhalte aus den medizinischen Grundlagenfächern könnten Psychotherapeuten jedoch die dazu erforderlichen Kompetenzen erwerben. Diese Zusatzqualifikation würde sicher nicht von allen PP und KJP angestrebt, da z.B. auch viele Fachärzte für Psychosomatische Medizin und Psychotherapie in der ambulanten Versorgung nahezu ausschließlich psychotherapeutisch tätig sind und die psychopharmakologischen Behandlungsoptionen nicht nutzen.

Die Frage, ob PP und KJP Aufgaben übernehmen können, die bislang Ärzten vorbehalten sind, kann also mit einem klaren Ja beantwortet werden. Aber ist es mit Blick auf die
Versorgung auch wünschenswert oder sogar notwendig, dass sie diese Tätigkeiten ausüben?

Die Medizin des 21. Jahrhunderts verlangt auch für die Versorgung psychisch kranker Menschen einen neuen Zuschnitt der Professionsgrenzen und ein modernes Kooperationsmodell jenseits der überkommenen Arztzentrierung. Während psychische Erkrankungen zu neuen Volkskrankheiten avancieren, muss die Ärzteschaft einen dramatischen Nachwuchsmangel beklagen, von dem die Fachärzte für Psychosomatische Medizin und Psychotherapie und die Fachärzte für Psychiatrie und Psychotherapie besonders betroffen sind. Gleichzeitig steigt mit zunehmender evidenzbasierter Ausrichtung der Versorgung psychisch kranker Menschen der Stellenwert der Psychotherapie in den Behandlungsempfehlungen evidenzbasierter Leitlinien, und Psychotherapie findet bei den Patienten eine immer höhere Akzeptanz. Diese positive Entwicklung in der Versorgung wird dadurch konterkariert, dass Patienten bei einer akuten Krise oder einer gravierenden Verschlechterung zwar notfallmäßig ihren behandelnden Psychotherapeuten kontaktieren und aufsuchen, für eine AU-Bescheinigung oder eine Einweisung ins Krankenhaus aber zusätzlich einen Arzt aufsuchen müssen, der mit der aktuellen Krankengeschichte meistens wenig vertraut ist.

Ein am Patientenbedarf und der Patientenpräferenz orientiertes evidenzbasiertes Behandlungsangebot braucht Behandler, die die Gesamtverantwortung übernehmen können. Zum Wohle der Patienten und im Sinne einer effizienten Nutzung der Ressourcen im Gesundheitswesen sollten daher die für die PP und KJP noch bestehenden Befugniseinschränkungen aufgehoben werden.

Johannes Klein-Heßling \& Timo Harfst, Berlin

\section{Contra}

\section{Durch Erweiterung der Kompetenzen von Psychologischen Psychotherapeuten könnten Parallelstrukturen entstehen, die einem ressourcenadaptierten, koordinativen Vorgehen entgegenwirken}

Dass die Verordnung von Psychopharmaka aus Gründen der hohen Verantwortung gegenüber Patientenschutzrechten ärztliche Expertise voraussetzt, das liegt auf der Hand. Psychologischen Psychotherapeuten (PP) wird weder im Studium noch in der Ausbildung zum Psychotherapeuten das pharmakologische Wissen über die Wirkmechanismen von Psychopharmaka vermittelt, vor allem lernen sie nicht die Nebenwirkungen und schädlichen Interaktionen mit anderen Pharmaka, die für Patienten eine erhebliche Gefährdung 
darstellen können. Dieser Punkt wird weitgehend gleich eingeschätzt: Psychologen wissen, dass sie im Falle von Fehlentscheidungen die möglichen juristischen Konsequenzen nicht tragen können, und sie wollen dies auch nicht.

Geteilt ist hingegen die Meinung dazu, ob PP Arbeitsunfähigkeitsbescheinigungen und Krankenhauseinweisungen ausstellen können und dies künftig auch dürfen sollen. PP erbringen für die Patienten häufig einen großen Beitrag zur Therapie, und die nicht unbegründeten Sorgen um die Versorgung der Patienten anlässlich zurückgehender Ärztezahlen in der stationären und ambulanten Versorgung fachen die Diskussion um die Kompetenzerweiterung von PP an.

Für die Allgemeinheit kostentreibende Maßnahmen wie Krankschreibungen und Krankenhauseinweisungen (letztere auch mit erheblichen Folgen für die psychosoziale Situation der Betroffenen) sollen aus wirtschaftlichen Gründen auf die Situationen beschränkt bleiben, in denen die ambulanten Behandlungsmöglichkeiten ausgeschöpft sind. Daraus ergibt sich, dass diese Entscheidungen von denen übernommen werden sollten, die über die Möglichkeiten der notwendigen Organdiagnostik und vor allem eines mehrdimensionalen Therapieansatzes aus Psychotherapie, Pharmakotherapie und Soziotherapie verfügen. Allein der Arzt kann aufgrund seines Wissens um alle Facetten des biopsychosozialen Bedingungsgefüges psychischer Erkrankungen den geeigneten Gesamtbehandlungsplan erstellen, die Behandlungsbausteine koordinieren und zu einer ausgewogenen Beurteilung kommen, ob eine Behandlungsform oder die Kombination mehrerer die Zielsymptome der gegebenen psychischen Störung in angemessener Zeit so weit verbessern können, dass eine Krankschreibung oder auch eine Krankenhauseinweisung vermeidbar ist. Schließlich ist auch zu bedenken, dass PP zur Notfallversorgung und Krisenintervention in der Versorgung nicht beitragen. Vielmehr erstreckt sich ihr Patientenkontakt im Rahmen der Richtlinienverfahren häufig auf einen sehr geregelten, einmal wöchentlichen Kontakt. Entscheidungen über die Weiterbehandlung in stationären Versorgungsformen müssen jedoch schneller getroffen werden.
Vor allem eine Krankenhausbehandlung darf nur erfolgen, wenn andere Behandlungsmöglichkeiten tatsächlich nicht ausreichen. Es dürfte kaum mit der gesundheitspolitischen Zielsetzung der Begrenzung kostenintensiver stationärer Behandlungen vereinbar sein, wenn die Messlatte für Einweisungen niedriger gehängt würde.

PP sollten an der Entscheidungsbildung für Krankschreibung und Krankenhausbehandlung teilnehmen, aber diese für Patienten und Kostenträger gleichermaßen wichtigen Entscheidungen müssen in einem vernetzten Versorgungssystem getroffen werden. Der Mediziner hat aufgrund der Breite seiner Kompetenz die Aufgabe, die verschiedenen Versorgungsebenen zusammenzuführen und bei diesen Entscheidungen abzuwägen. Entsprechend dem Gedanken der Vernetzung der an der Versorgung beteiligten Berufsgruppen wäre die Tätigkeit von PP verstärkt in vernetzten Strukturen zu konzeptionalisieren, und es wären nicht im Gegenteil Wege zu bahnen, die einer solchen Vernetzung zuwiderlaufen. Durch Erweiterung der Kompetenzen von PP, so muss befürchtet werden, könnten Parallelstrukturen entstehen, die einem ressourcenadaptierten, koordinativen Vorgehen entgegenwirken.

Bereits im Studium, vor allem aber im Rahmen der Ausbildung zum PP werden medizinische Grundkenntnisse vermittelt. Diese Ausbildung ist darauf gerichtet, eine Kompetenz dafür zu entwickeln, in welchen Situationen Ärzte zur differenzialdiagnostischen Klärung oder zur Einleitung einer zusätzlichen pharmakotherapeutischen Intervention hinzuzuziehen sind. Ohne medizinisches Studium können im Rahmen der «Praktischen Tätigkeit» nicht die notwendigen pathophysiologischen, neuroanatomischen, pharmakologischen und auch nicht die notwendigen sozialmedizinischen Kenntnisse erworben werden, um den möglichen Erfolg, der durch die Kombination von Psychotherapie mit weiteren therapeutischen Maßnahmen auch unter ambulanten Bedingungen eintreten könnte, abschätzen zu können. Deshalb sollten auch künftig die Verordnung von Medikamenten, Arbeitsunfähigkeitsbescheinigungen, Krankenhauseinweisungen und die Verschreibung von Hilfsmitteln in einer, nämlich der ärztlichen Hand bleiben.

Sabine Herpertz, Heidelberg 